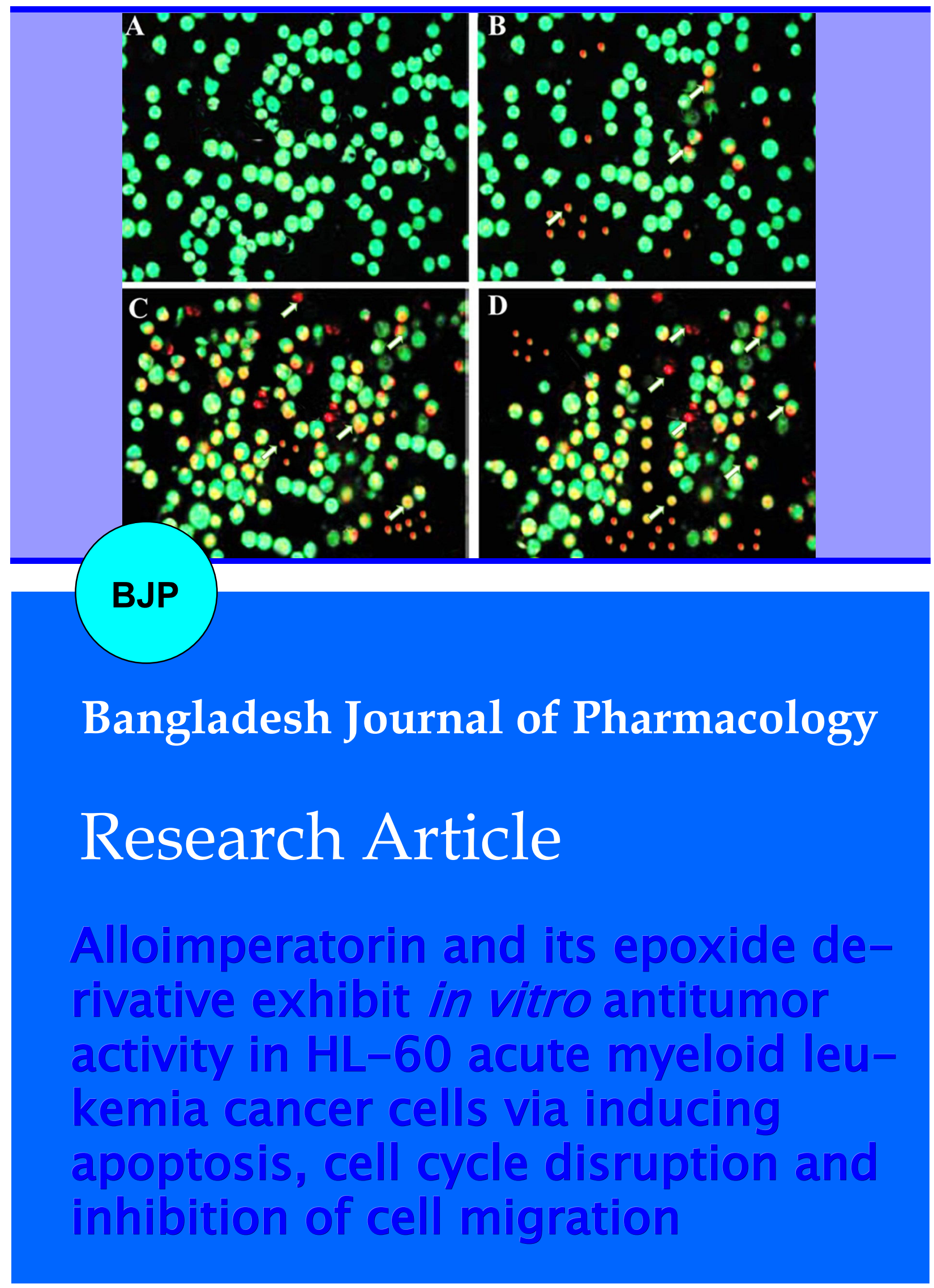


Abstracted/indexed in Academic Search Complete, Asia Journals Online, Bangladesh Journals Online, Biological Abstracts, BIOSIS Previews, CAB Abstracts, Current Abstracts, Directory of Open Access Journals, EMBASE/Excerpta Medica, Google Scholar, HINARI (WHO), International Pharmaceutical Abstracts, Open J-gate, Science Citation Information Expanded (SCIE), SCOPUS and Social Sciences Citation Index;

ISSN: $1991-0088$

\title{
Alloimperatorin and its epoxide derivative exhibit in vitro antitumor activity in HL-60 acute myeloid leukemia cancer cells via inducing apoptosis, cell cycle disruption and inhibition of cell migration
}

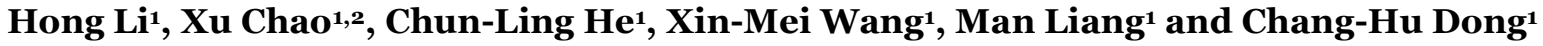 \\ ${ }^{1}$ Department of Hematology, the Second Affiliated Hospital of Shanxi, University of Chinese Medicine, Xianyang \\ 712 000, China; ${ }^{2}$ The College of Preclinical Sciences, Shanxi University of Chinese Medicine, Xianyang 712046 , \\ China.
}

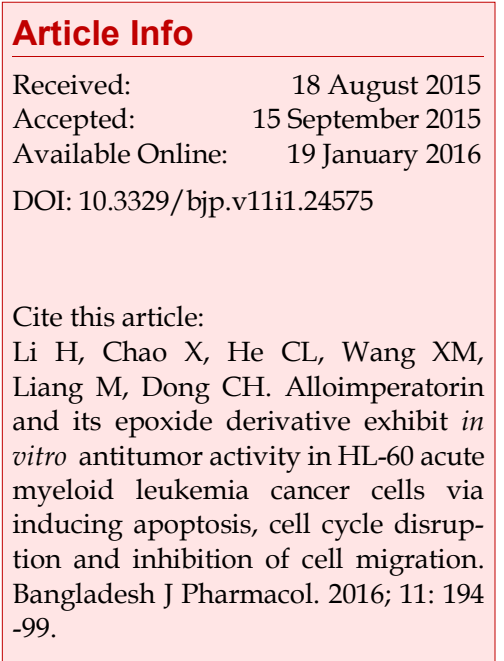

\begin{abstract}
The aim of the present study was to synthesize epoxide derivative of alloimperatorin and evaluating its antitumor and apoptotic effects in acute myeloid leukemia HL-60 cells. The cytotoxic effects were demonstrated by MTT assay. Fluorescence microscopy along with flow cytometry were performed to evaluate the effect of alloimperatorin epoxide on apoptosis and cell cycle. In vitro wound healing assay was performed to study compound's effect on cancer cell migration. The results indicated that alloimperatorin epoxide $\left(\mathrm{IC}_{50}=32.1 \mu \mathrm{M}\right)$ was much more effective in inhibiting HL-60 cancer cell growth as compared to alloimperatorin $\left(\mathrm{IC}_{50}=128 \mu \mathrm{M}\right)$. Further, alloimperatorin epoxide induced apoptosis related morphological alterations in HL-60 cells including blebbing of plasma membrane, DNA fragmentation and formation of apoptotic bodies. Alloimperatorin epoxide also led to G2/M phase cell cycle arrest and suppressed HL-60 cancer cell migration indicating that this compound may be a promising candidate for the treatment of cancer metastasis.
\end{abstract}

\section{Introduction}

Leukemia is a collection of cancers that frequently initiates in the bone marrow and leads to high numbers of defunct white blood cells (Hoffman, and Ronald, 2005). Acute myeloid leukemia (AML) is a tumor of the myeloid line of blood cells. AML is the most common acute leukemia in adults with its prevalence rate increasing with age. AML accounts for only $1.2 \%$ of cancer related deaths in USA, but it is estimated that its incidence rate will increase as the population ages (Jemal et al., 2002). Leukemia ranks as the seventh most common malignant cancer prevailing in China accounting for an incidence rate of 2.76/10 million (Yang and Zhang, 1991). Despite the latest developments in clinical therapy of leukemia, the overall effectiveness of the treatment process remains very poor. Conventional chemotherapeutic agents like paclitaxel and doxorubicin kill cancer cells by facilitating cell apoptosis or necrosis process. However, they also target and kill normal cells and as such result in serious adverse effects which is the reason why these chemotherapeutic agents have limited efficacy (Friesen et al., 1996).

The antileukemic potential of natural plant based chemotherapeutic agents have gained attention and significant research work has been performed in order to isolate and characterize these compounds and demonstrate their molecular mechanism of action. (Durgo et al., 2013; Ravishankar et al., 2013; Gach and Janecka, 2014). The objective of the current study was to demonstrate the antitumor efficacy of alloimperatorin and its synthesized epoxide derivative in HL-60 acute 
myeloid leukemia cancer cells.

\section{Materials and Methods \\ Chemicals and other reagents}

Alloimperatorin (>98\%) was purchased from Chengdu Preferred Biotech Co. Ltd (China) and was dissolved in dimethyl sulfoxide at the concentration of $20 \mathrm{mM}$, stored as small aliquots at $-20^{\circ} \mathrm{C}$. Different concentrations of the compound were prepared as needed in cell culture experiments. Propidium iodide, trypsin and dimethyl sulfoxide were purchased from Sigma-Aldrich (USA). 3-[4,5-dimeth-yl-2-thiazolyl]-2,5-diphenyl tetrazolium bromide (MTT), was purchased from Molecular Probes (USA). Dulbecco's modified Eagle's medium, fetal bovine serum (FBS), penicillin-streptomycin and acridine orange were obtained from Hangzhou Sijiqing Biological Products Co. Ltd. China.

\section{Synthesis and spectral characterization of alloimpera- torin epoxide}

Epoxide of alloimperatorin (B) was obtained by the addition of m-chloroperbenzoic acid $(20 \mathrm{~mL})$ to alloimperatorin (A) solution (200 $\mathrm{mg}$ ) in dichloromethane under cold conditions (Figure 1). After accomplishment, the reaction mixture was diluted with water and extracted with chloroform. The organic layer was dried by using $\mathrm{Na}_{2} \mathrm{SO}_{4}$ and purified through column chromatography to furnish compound (B) in extra pure form. Compound B showed a prominent peak in IR spectrum at $775 \mathrm{~cm}^{-1}$, characteristic of trisubstituted epoxide, besides other bands at $1730 \mathrm{~cm}-1,1605$ and $1280 \mathrm{~cm}-1$. In proton spectrum, the chemical shift and multiplicity at C-2' supports the structure. ${ }^{13} \mathrm{C}-\mathrm{NMR}$ and mass spectrum confirmed the identity of the compound.

\section{Cell Line and culture medium}

HL-60 human acute myeloid leukemia cell line was procured from the Cancer Research Institute of Beijing, China. The cells were grown in DMEM supplemented with $10 \%$ fetal calf serum (FBS) and 150 units $/ \mathrm{mL}$ of penicillin. Incubation of the cells was done at $37^{\circ} \mathrm{C}$ in a humidified atmosphere of $5 \% \mathrm{CO}_{2}$ and $95 \%$ air. The media was stored at low temperature $\left(2-5^{\circ} \mathrm{C}\right)$.

\section{MTT assay for cell viability}

HL-60 cells were seeded at a density of $2 \times 10^{5}$ cells/ well and cultured with different doses of alloimperatorin and alloimperatorin epoxide $(0,4,16,32,64$ and $128 \mu \mathrm{M}$ each) for 24 hours. After drug treatment, the medium was changed and 3-(4,5-dimethylthiazol-zyl)2,5-diphenyltetrazolium bromide (MTT: $1.5 \mathrm{mg} / \mathrm{mL}$ ) was added for 5 hours. The fraction of the viable cells is equal to the formation of formazan crystals which were dissolved in ethanol and the optical density was measured spectrophotometrically at $565 \mathrm{~nm}$. The number of viable cells was calculated by comparing with the untreated control cell line.

\section{Fluorescence microscopy assay using acridine orange and propidium iodide staining}

HL-60 cells $\left(2 \times 10^{5}\right.$ cells $\left./ \mathrm{mL}\right)$ were taken in a petri dish and treated with $0,4,32$ and $128 \mu \mathrm{M}$ of alloimperatorin epoxide for 48 hours. After drug treatment, cells were washed with PBS, and then stained with acridine orange $(5 \mu \mathrm{g} / \mathrm{mL}$ in PBS) and propidium iodide $(5 \mu \mathrm{g} /$ $\mathrm{mL}$ in PBS) for $20 \mathrm{~min}$ at $37^{\circ} \mathrm{C}$. $20 \mu \mathrm{L}$ of the cell suspension was taken on a slide and images were captured using a fluorescence microscope (magnification, x400; Nikon, Japan).

\section{Cell cycle analysis by flow cytometry}

The cell cycle analysis was performed by FACS Calibur instrument (BD Biosciences, USA, equipped with Cell Quest 3.3 software with DNA propidium iodide staining. HL-60 cells $\left(2 \times 10^{5}\right.$ cells $\left./ \mathrm{mL}\right)$ were seeded in $60-\mathrm{mm}$ dishes and treated with $0,4,32$ and $128 \mu \mathrm{M}$ of alloimperatorin epoxide for 48 hours. After drug treatment, the cells were trypsinized and washed twice with PBS. After that the cells were fixed with $70 \%$ cold ethanol overnight and then treated with $10 \mu \mathrm{g} / \mathrm{mL}$ RNase A, then stained with $2.5 \mu \mathrm{g} / \mathrm{mL}$ of propidium iodide. Finally the DNA content and cell cycle distribution was analyzed by flow cytometry. The experiments were repeated three times.

\section{Cell migration assay}

This assay was done according to a standard method already reported (Liang et al., 2007). HL-60 cells ( $2 \times 10^{5}$ cells $/ \mathrm{mL}$ ) were seeded in a 6-well plate and incubated at $37^{\circ} \mathrm{C}$ until $90-95 \%$ full confluent monolayer of cells was obtained. A $100 \mathrm{~mL}$ pipette tip was used to make a straight cell-free wound after 12 hours of starvation. Each well was washed three times with PBS to remove any cellular debris and then subjected to several doses of alloimperatorin epoxide $(0,4,32$ and $128 \mu \mathrm{M})$ in a medium. After 48 hours of incubation, the cells were fixed and stained with 5\% ethanol containing 0.5\% crystal violet powder for $20 \mathrm{~min}$, and randomly chosen fields were photographed under a light microscope (inverted light microscope IX71; Olympus, Japan). The number of cells that migrated into the scratched area were counted and lengths of wound were determined by Image J (version 1.46) software.

\section{Statistical analysis}

Data are presented as the mean \pm SEM of the control. All experiments were repeated at least three times. The differences between groups were analyzed by one-way ANOVA with Tukey's posthoc tests, significance of difference was indicated as ${ }^{*} \mathrm{p}<0.05,{ }^{* *} \mathrm{p}<0.01$. 


\section{Results}

Cytotoxic activity of alloimperatorin and alloimperatorin epoxide in HL-60 leukemia cells

The results of the current study indicated that alloimperatorin epoxide was much more effective in inhibiting the growth of HL-60 cancer cells as compared to the alloimperatorin (Figure 1). The $\mathrm{IC}_{50}$ values of alloimperatorin and alloimperatorin epoxide were calculated to be $128 \mu \mathrm{M}$ and $32.1 \mu \mathrm{M}$ respectively. This indicates that the epoxide of alloimperatorin was much more potent in inhibiting the cancer cell growth of HL60 cancer cells (Figure 2). For this reason, further in depth biological assays were performed only on alloimperatorin epoxide rather than alloimperatorin.

\section{Effects of alloimperatorin epoxide on the cellular morphology and apoptosis}

Fluorescence microscopy using acridine orange and propidium iodide staining indicated that alloimperatorin epoxide induced morphological changes characteristic of apoptosis including chromatin condensation, plasma membrane blebbing, apoptotic body formation and fragmentation of DNA. The results of acridine orange/ propidium iodide staining showed that as compared to the control group (Figure 3A), alloimperatorin epoxidetreated cells revealed chromatin condensation and nuclear fragmentation (nuclei with red colour). The number of cells with red color increase as the concentration of compound increases (Figure 3B-D). The process of apoptosis is characterized by various morphological alterations which were observed after alloimperatorin epoxide treatment.

\section{Effect of alloimperatorin epoxide on cell cycle phase distribution and DNA content}

Cell cycle process and cell proliferation are intimately related events. In order to demonstrate whether alloimperatorin affects cell cycle of HL-60 leukemia cells, the cell cycles were examined by flow cytometry using

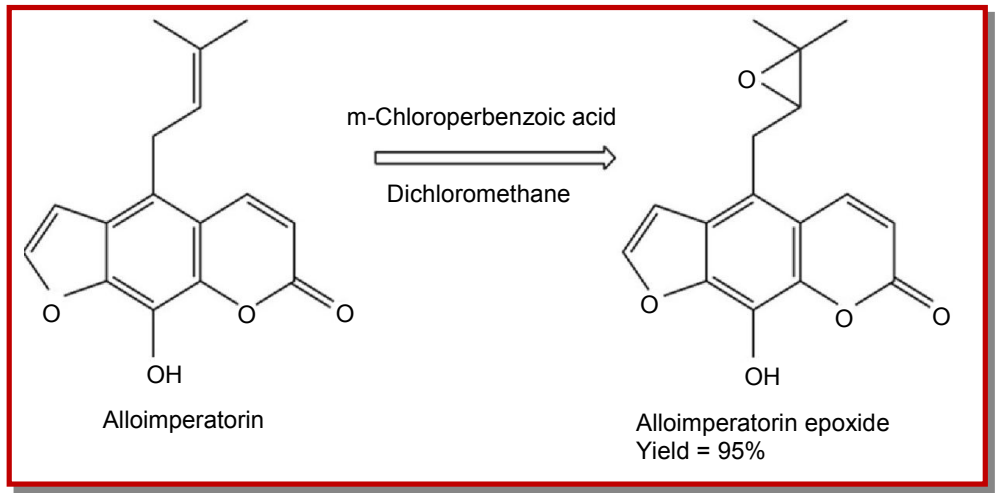

Figure 1: Synthetic procedure for the synthesis of epoxide derivative of alloimperatorin using m-chloroperbenzoic acid in dichloromethane

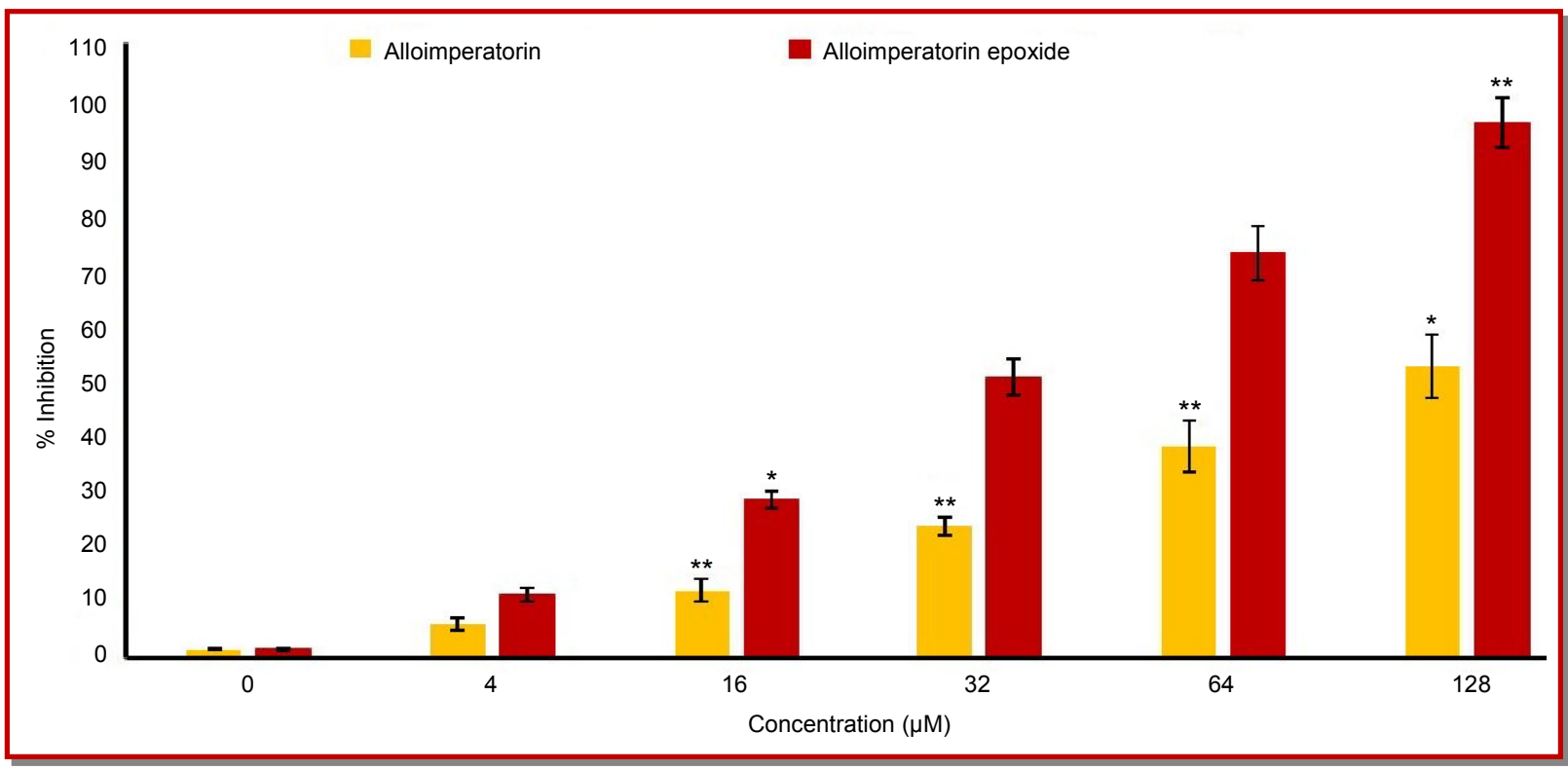

Figure 2: Cytotoxic effect of alloimperatorin and alloimperatorin epoxide in human leukemia cells (HL-60). Data are shown as the mean \pm SD of three independent experiments. ${ }^{*} \mathrm{p}<0.05$, ${ }^{* *} \mathrm{p}<0.01$, vs $0 \mu \mathrm{M}$ (control) 
FACS Calibur instrument (BD Biosciences, USA). The findings of the study revealed that alloimperatorin led to an increase in the G2/M cell population while as the number of G0/G1 cells experienced a sharp decrease as the concentration of the compound was increased from $0 \mu \mathrm{M}$ to 4,32 and $128 \mu \mathrm{M}$ (Figure 4). The percentage of G2/M cells increased from $12.1 \%$ in untreated cells to $14.5 \%, 38.7 \%$ and $67.2 \%$ in 4,32 and $128 \mu \mathrm{M}$ alloimperatorin epoxide-treated cells respectively.

\section{Alloimperatorin epoxide suppresses HL-60 cancer cell migration}

Highly invasive malignant tumors are characterized by enhanced migratory tendency. As such, suppression of cancer cell migration could be an effective strategy to thwart tumor metastasis. In the present study, we investigated whether alloimperatorin could result in inhibition of cancer cell migration in HL-60 cells. Different doses of the compound were used and the percentage of cells that migrated were calculated. The results of this study revealed that alloimperatorin epoxide significantly inhibited wound closure and it was observed that $32 \mu \mathrm{M}$ dose of the compound inhibited wound closure by $56.3 \%$ as compared to the vehicle control (Figure 5).

\section{Discussion}

The aim of the study was to design and synthesize the epoxide derivative of alloimperatorin and evaluate its anticancer and apoptotic effects against acute myeloid leukemia cells (HL-60). The MTT results revealed that both alloimperatorin and epoxide derivative showed anticancer activity but the latter was much more effective as compared to the former. The $\mathrm{IC}_{50}$ values alloimperatorin and alloimperatorin epoxide were calculated to be $128 \mu \mathrm{M}$ and $32.1 \mu \mathrm{M}$ respectively. Further, fluorescence microscopy using acridine orange and propidium iodide staining revealed that alloimpera-
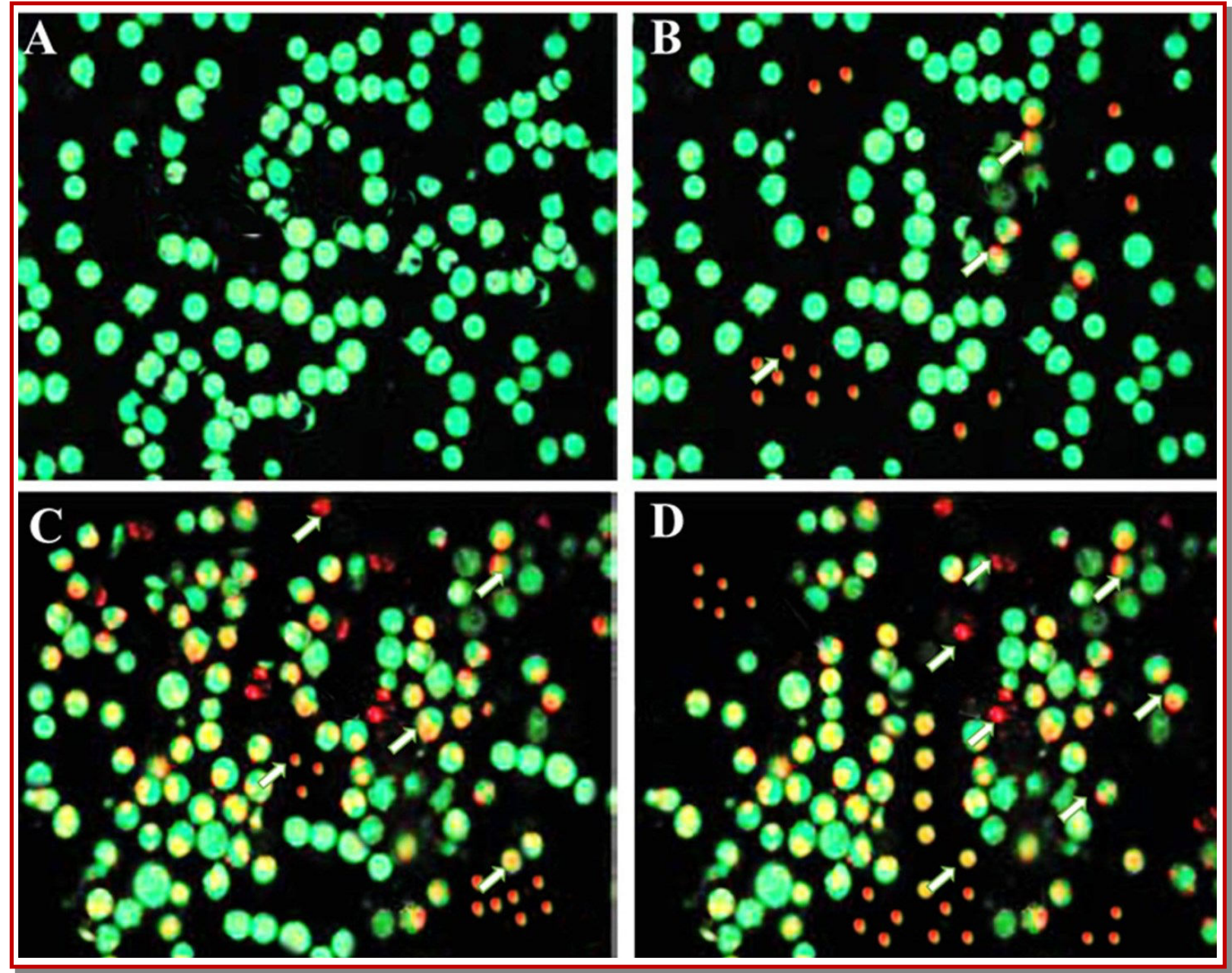

Figure 3: Fluorescence microscopic study of HL-60 cells using acridine orange/propidium iodide staining method. A, represents untreated control, B, C and D, represent HL-60 cells treated with 4, 32 and $128 \mu \mathrm{M}$ dose of alloimperatorin epoxide respectively. The apoptotic cells (red fluorescence) indicated chromatin condensation or chromatin fragmentation (white arrows). The number of apoptotic cells (red color) increases with increase in alloimperatorin dose 


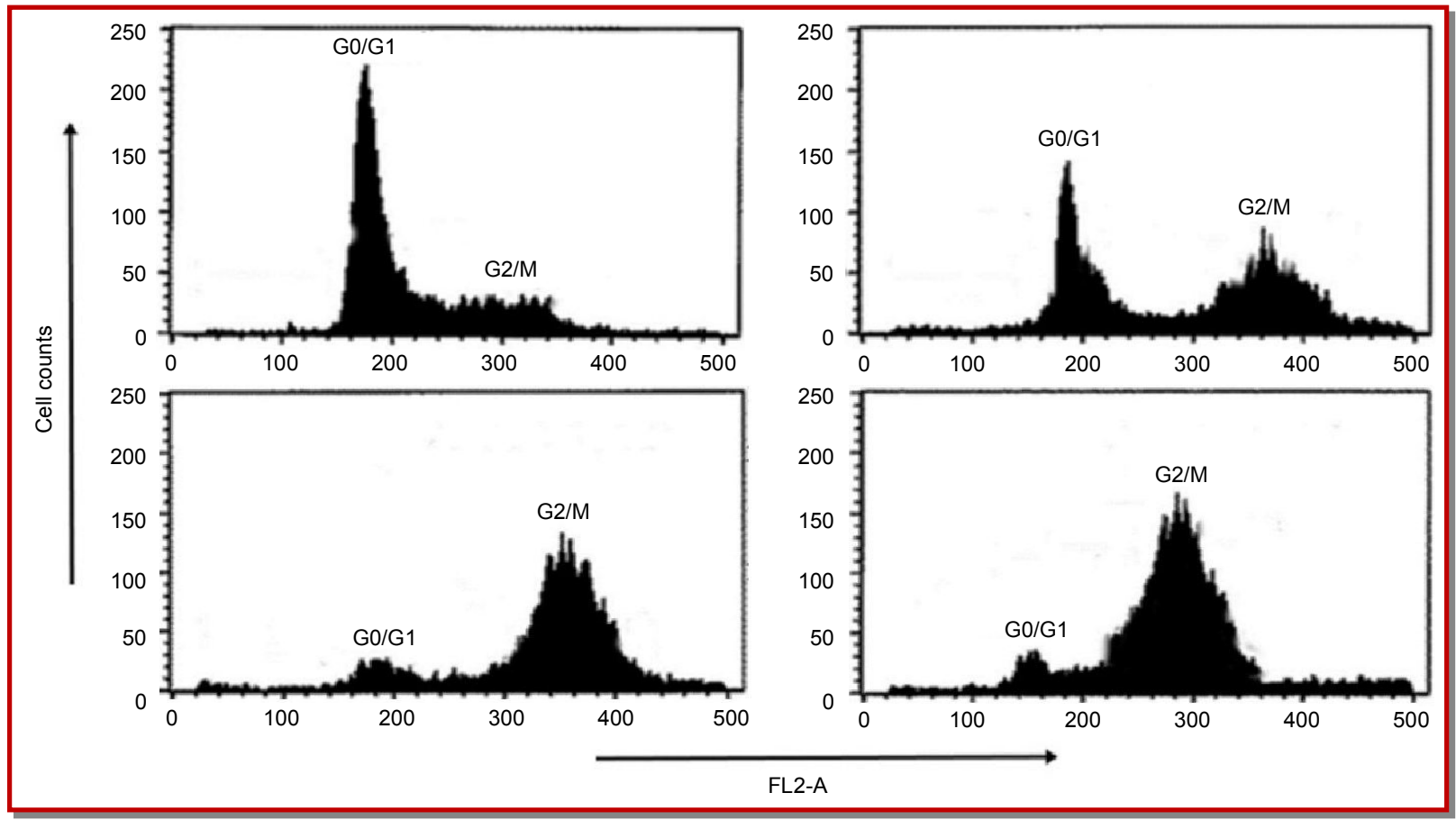

Figure 4: Effect of alloimperatorin epoxide on cell cycle progression in HL-60 human leukemia cells. As shown, alloimperatorin induced G2/M phase cell cycle arrest which showed strong dose-dependence. A represents untreated control, B, C and D represent effect of 4, 32 and $128 \mu \mathrm{M}$ dose of alloimperatorin epoxide on cell cycle phase distribution. The cell cycle experiment was performed using FACS Calibur flow cytometer. The data presented here are representative of at least three separate experiments

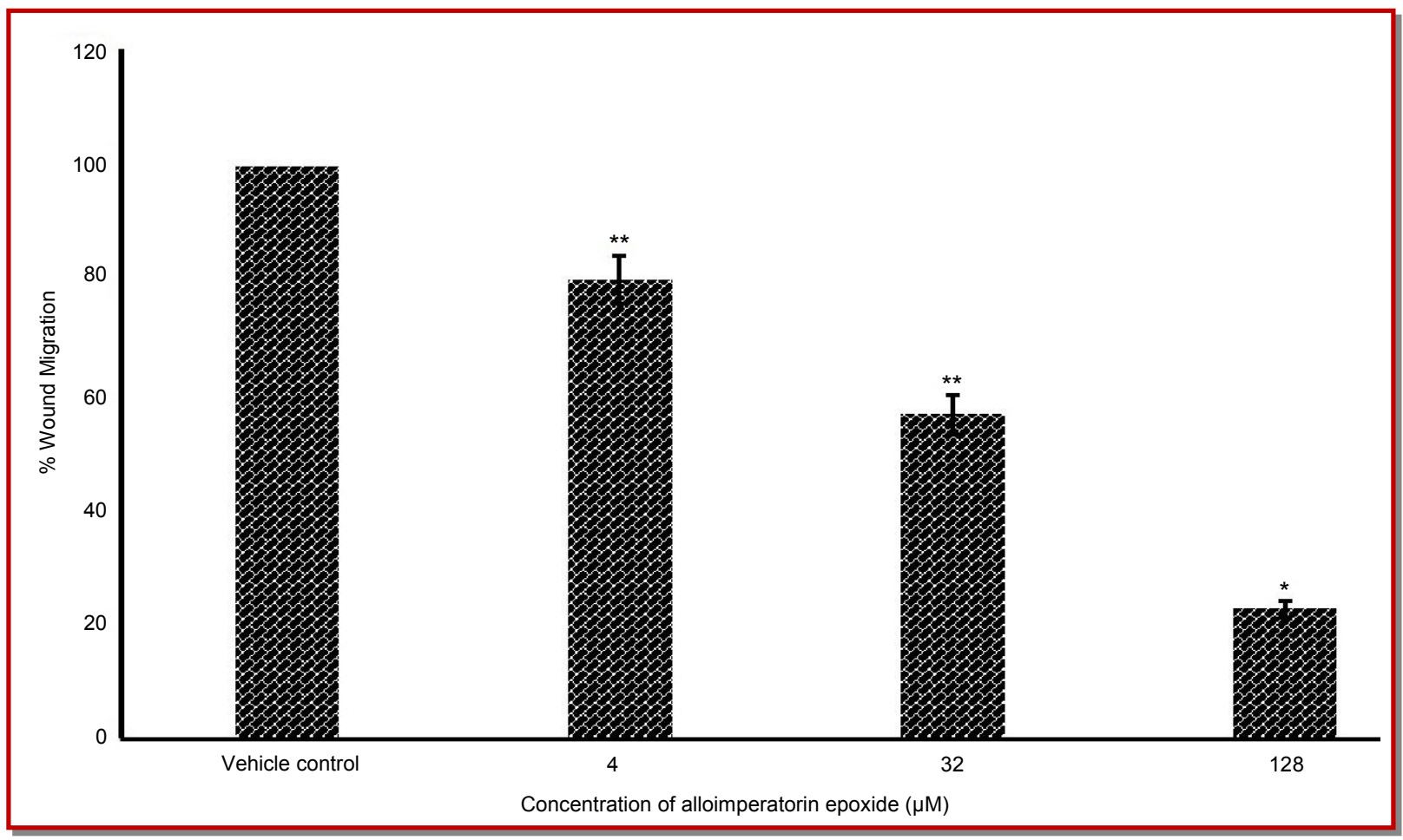

Figure 5: Alloimperatorin epoxide inhibits HL-60 cancer cell migration. The cells were treated with 0, 4, 32 and $128 \mu \mathrm{M}$ dose of alloimperatorin epoxide for 48 hours and photographs were captured using inverted light microscope. The number of migrated cells were calculated and shown graphically as above. ${ }^{*} \mathrm{p}<0.05$, ${ }^{* *} \mathrm{p}<0.01$, vs $0 \mu \mathrm{M}$ (control) 
torin epoxide induced apoptosis-related morphological changes in HL-60 cells including blebbing of plasma membrane, DNA fragmentation and formation of apoptotic bodies (red fluorescence). The number of cells with red colour increase as the concentration of compound increases. Flow cytometry revealed that alloimperatorin epoxide led to G2/M phase cell cycle arrest in HL-60 cells which showed strong dose-dependence. In vitro wound healing assay revealed that alloimperatorin epoxide resulted in inhibition of HL-60 cancer cell migration and as such this compound can be a promising candidate for the treatment of tumor metastasis.

Alloimperatorin (9-hydroxy-4-(3-methyl-2-buten-1-yl)$7 \mathrm{H}$-furo[3,2-g]chromen-7-one) is a naturally occurring coumarin compound found in many medicinal plants including Conyza canadensis and Aegle mermelos (Manjunatha and Amith, 2011). Not enough research work has been reported on alloimperatorin so far. However, several reports have been published on imperatorin, which is a close structural analogue of alloimperatorin. Imperatorin has been reported to inhibit HepG2 cancer cell proliferation through apoptosis induction, alteration in cellular morphology, DNA fragmentation, phosphatidylserine externalization and loss of mitochondrial membrane potential (Luo et al., 2011). Imperatorin has also been shown to affect cognitive behavior and oxidative stress in the brain induced by scopolamine in the male Swiss mice. This compound has been shown to inactivate gamma-aminobutyric acid transaminase and inhibits acetylcholinesterase activity.

Our present study on the epoxide derivative of alloimperatorin on HL-60 leukemia cells is a first such report. Inhibiting the unceasing growth of tumor cells is an effective mechanism to control cancer. It has been reported that G2/M phase of the cell cycle constitutes a very crucial stage for controlling the cell division. There are various agents which target this key stage of cell cycle and are effective chemotherapeutic agents in cancer therapy. But, there are very few chemotherapeutic agents which have been found to affect G2/M phase. In the present study, alloimperatorin epoxide derivative has been found to affect G2/M cell cycle phase and as such cause G2/M cell cycle arrest (Hsu et al., 2011; Xu et al., 2011; Wilkinson et al., 2007).

\section{Conclusion}

Alloimperatorin epoxide suppresses proliferation of HL -60 acute myeloid leukemia cells by inducing apoptosis, G2/M cell cycle arrest and inhibition of cell migration.

\section{Conflict of Interest}

The authors declare that there is no conflict of interest to reveal.

\section{References}

Durgo K, Koncar M, Komes D, Belscak-Cvitanovic A, Franekic J, Jakopovich I, Jakopovich N, Jakopovich B. Cytotoxicity of blended versus single medicinal mushroom extracts on human cancer cell lines: Contribution of polyphenol and polysaccharide content. Int J Med Mushrooms. 2013; 15: 43548.

Friesen C, Herr I, Krammer PH, Debatin KM. Involvement of the CD95 (APO-1/FAS) receptor/ligand system in druginduced apoptosis in leukemia cells. Nat Med. 1996; 2: 57477.

Gach K, Janecka A. a-Methylene- $\gamma$-lactones as a novel class of anti-leukemic agents. Anticancer Agents Med Chem. 2014; 14: 688-94.

Hoffman, Ronald. Hematology: Basic principles and practice. 4th ed. St. Louis, Elsevier Churchill Livingstone, 2005, pp 1074-75.

Hsu HF, Huang KH, Lu KJ, Chiou SJ, Yen JH, Chang CC, Houng JY. Typhonium blumei extract inhibits proliferation of human lung adenocarcinoma A549 cells via induction of cell cycle arrest and apoptosis. J Ethnopharmacol. 2011; 135: 492-500.

Jemal A, Thomas A, Murray T, Thun M. Cancer statistics, 2002. CA Cancer J Clin. 2002; 52: 23-47.

Liang CC, Park AY, Guan JL. In vitro scratch assay: A convenient and inexpensive method for analysis of cell migration in vitro. Nat Protoc. 2007; 2: 329-33.

Luo KW, Sun JG, Chan JY, Yang L, Wu SH, Fung KP, Liu FY. Anticancer effects of imperatorin isolated from Angelica dahurica: Induction of apoptosis in HepG2 cells through both deathreceptor- and mitochondria-mediated pathways. Chemotherapy 2011; 57: 449-59.

Manjunatha BK, Amith RR. Alloimperatorin from Aegle mermelos; A promising lead molecule for hepatoprotection: In vivo and in silico approach. Int J Curr Res. 2011; 3: 5-10.

Ravishankar D, Rajora AK, Greco F, Osborn HM. Flavonoids as prospective compounds for anti-cancer therapy. Int J Biochem Cell Biol. 2013; 45: 2821-31.

Wilkinson RW, Odedra R, Heaton SP, Wedge SR, Keen NJ, Crafter C, Foster JR, Brady MC, Bigley A, Brown E, Byth KF, Barrass NC, Mundt KE, Foote KM, Heron NM, Jung FH, Mortlock AA, Boyle FT, Green S. AZD1152, a selective inhibitor of Aurora B kinase, inhibits human tumor xenograft growth by inducing apoptosis. Clin Cancer Res. 2007; 13: 3682-88.

Xu X, Zhang Y, Qu D, Jiang T, Li S. Osthole induces G2/M arrest and apoptosis in lung cancer A549 cells by modulating PI3K/Akt pathway. J Exp Clin Cancer Res. 2011; 30: 33.

Yang C, Zhang X. Incidence survey of leukemia in China. Chin Med Sci J. 1991; 6: 65-70.

Author Info

I Chang-Hu Dong (Principal contact)

e-mail: dongchanghu19@hotmail.com

| First two authors contributed equally 


\section{Your feedback about this paper}

1. Number of times you have read this paper 0

2. Quality of paper Click

3. Your comments 